\title{
MODIFICATIONS DANS LA STRUCTURE DE LA PROPRIÉTÉ PRIVÉE EN FINLANDE*
}

\author{
M. A. REUNALA
}

Class. Oxford $923(471.1)$

Cette thèse d'économie forestière a comme objet principal l'étude des mutations en cours au sein du groupe social des propriétaires privés finlandais.

La situation décrite n'a pour le lecteur français qu'un intérêt documentaire. C'est la tentative pour construire un outil d'analyse qui doit retenir l'attention. Il s'agit, en effet, d'un remarquable effort pour mettre au point une méthode rationnelle de réflexion dans un domaine peu exploré et dont l'intérêt économique est mis en lumière.

\section{IMPORTANCE DE LA FORÊT ET DES INDUSTRIES DU BOIS}

Les forêts productives couvrent $61 \%$ de la surface de la Finlande et constituent la matière première de la plus importante branche industrielle du pays qui assure à elle seule $50 \%$ des exportations. Toute la ressource provenant de la forêt est déjà mobilisée, de telle sorte que l'expansion des ventes de produits finis suppose une progression préalable du croit des peuplements.

C'est donc un impératif national que de développer les ressources forestières afin d'alimenter un courant d'exportations qui a bien du mal à compenser la masse sans cesse croissante des importations.

\section{STRUCTURE DE LA FORÊT PRIVÉE}

$65 \%$ de la forêt finlandaise appartient à des particuliers. La part de ce type de propriétaire dans le volume sur pied et dans l'accroissement de la totalité des forêts du pays est encore plus élevée.

C'est dire à quel point l'économie finlandaise est tributaire de l'évolution de la forêt particulière.

Cette dernière ayant une structure très différente de celle que nous connaissons en France, quelques éclaircissements s'imposent.

Indiquons d'abord que le relief en " peau d'orange " d'une grande partie de la Finlande ne rend apte à l'agricuiture que des surfaces disjointes séparées par des espaces en pente où le rocher affleure et qui portent la forêt.

L'exploitation rurale classique comprend un noyau agricole de 6 à 10 ha de terres et prés, entouré de 25 à 40 ha de bois.

(·) REUNALA (Aarne). - Structural change of private forest ownership in Finland. Doctoral dissertation, Helsinki, december 20th, 1974. - Helsinki, 1974 (Communicationes instituti forestalis fenniae 82. 2) 
Les exploitants sont propriétaires de leur fonds depuis les mouvements sociaux du début du siècle qui ont fait éclater les grands domaines au profit de tenanciers qui menaient jusque là une vie difficile.

II n'existait en 1969 pas moins de 350000 propriétaires forestiers particuliers qui, pour la plupart étaient de petits agriculteurs, fils de métayers devenus maîtres chez eux par éviction de leurs anciens bailleurs.

En raison de l'exiguité de telles structures, les agriculteurs ont un besoin impératif de tirer de leurs bois un revenu qui doit s'ajouter tous les ans à celui, bien modeste, de leur terre ou de leur troupeau.

Très vulnérables du fait de l'étroitesse de leur fonds, ils acceptent facilement l'encadrement d'organisations publiques rappelant quelque peu, mais avec des pouvoirs et des moyens de soutien très accrus, nos Centres régionaux de la propriété forestière et qui sont désignés en anglais sous le nom de "Forestry boards", et en français comme les « commissions forestières $\|$.

Les «forestry boards " sont à même de jouer le rôle de courroie de transmission entraînant les propriétaires dans la voie d'une sylviculture plus intensive dont la nécessité s'impose aux pouvoirs publics.

\section{LE PROBLEME}

Cette politique d'expansion de la ressource, tout comme le ravitaillement régulier des industries forestières, sont menacés par l'évolution de la structure même de la propriété particulière.

L'exode rural draine vers les villes les enfants des agriculteurs-sylviculteurs. La forêt passe progressivement aux mains de citadins qui se comportent de façon toute différente de celle de leurs aînés.

A la forêt "moyen de subsistance " se substitue la forêt " patrimoine " et les réactions des nouveaux propriétaires semblent surprendre les pouvoirs publics. On s'inquiète de voir toute la chaîne des industries de transformation dépendre du choix de personnes qui n'ont plus un besoin vital de récolter tous les ans le croît de leurs bois puisque la vie de leur famille est assurée par d'autres activités exercées loin de la terre.

L'étude menée par A. Reunala à partir de ce constat d'ensemble consiste en une estimation de l'importance et de la vitesse des transferts de surfaces de la "forêt de subsistance » à la " forêt patrimoine ".

\section{Elle se développe en trois étapes :}

- Recueil des informations relatives à la propriété particulière et aux surfaces en jeu.

- Description et analyse des changements qui se sont produits dans les années passées.

- Étude prospective devant fournir des éléments concrets utilisables pour la prise de décision en matière de politique forestière.

\section{RECUEIL DES INFORMATIONS}

L'auteur a fait exécuter une enquête par sondage portant sur la population des propriétaires de forêts dans une province jugée représentative de l'ensemble du pays. Un questionnaire 
très détaillé fut préparé et soumis à 510 propriétaires constituant un échantillon représentatif de l'ensemble de la profession dans la province.

Chaque personne interrogée était invitée à faire connaître non seulement l'étendue de ses bois et les caractéristiques générales de ses activités professionnelles et de son mode de vie personnel, mais également tout ce qui concernait la façon dont elle était devenue propriétaire, et les jugements de valeur qu'elle portait sur le mode de vie rural ou citadin, les motifs d'un éventuel départ vers la ville, la satisfaction ou les regrets résultant des choix opérés antérieurement.

\section{ANALYSE DES RÉSULTATS}

Les données collectées grâce à cette enquête permettent à l'auteur de dresser un tableau de l'évolution au cours des années passées de la propriété forestière privée. En 1970, $20 \%$ de la forêt privée était déjà entre les mains de non agriculteurs, l'héritage constituant le processus le plus courant dans ce glissement de la forêt subsistance vers la forêt patrimoine. Le phénomène allait en s'amplifiant.

\section{RECOURS A LA DYNAMIQUE DES SYSTĖMES}

La démarche centrale de l'étude consiste à proposer aux responsables de la politique forestière de meilleures méthodes de prévision en ce qui concerne l'évolution de la structure sociale de la propriété forestière.

Ceci est l'occasion pour l'auteur d'examiner les différentes voies utilisées en matière de prévision à long terme. II décrit le procédé de prolongement des courbes de tendances, la prospective des "horizons », la planification, et montre leur médiocre adaptation au problème étudié. C'est à la " dynamique des systèmes " qu'il est en fin de compte fait appel. Cette théorie a acquis une certaine notoriété à la suite des fameux rapports du Club de Rome qui l'utilise largement.

L'auteur décrit un "système » de la propriété forestière particulière en Finlande qui regroupe comme éléments constitutifs, non seulement les propriétés individuelles, mais aussi les " états " sous lesquels elles se présentent et les flux qui résultent du passage d'un état dans un autre. Pour les besoins de son analyse il ne considère que deux états : la propriété particulière de subsistance et la propriété patrimoine.

Le "Système » de la propriété forestière particulière est un système " ouvert " car il reçoit des impulsions de l'environnement dans lequel il est placé et en retour exerce un effet sur lui.

Les sorties du systeme sont :

- tel ou tel type de sylviculture,

- tel niveau des sorties de bois des forêts vers les industries utilisatrices, paysage.

- tel aspect de l'espace rural : le comportement des propriétaires s'inscrit dans le

La prise de conscience d'une corrélation entre l'état du système et les sorties - c'est-à-dire la diminution et l'irrégularité des sorties de bois des forêts patrimoines, est à l'origine de toute la démarche.

Les entrées du système sont plus délicates à définir. Elles proviennent de l'environnement dans lequel on peut discerner d'autres "systèmes " que celui qui vient d'être isolé. En particulier le "système social " général de la Finlande.

Les systèmes sont hiérarchisés les uns par rapport aux autres. Il est clair que le système social général est dominant par rapport au système de la propriété forestière. 
Le système social général définit les règles qui permettent les changements d'état à l'intérieur du système de la propriété forestière : héritage, transferts entre vifs. Les valeurs et normes sociales évoluent, ce que traduit au fur et à mesure la législation du pays. Cette évolution transforme et transformera les règles de changement d'état à l'intérieur de la propriété forestière privée.

Le système social lui-même est un système ouvert. Son évolution est avant tout commandée par une tendance très forte vers une urbanisation de plus en plus complète. En peu de décades, le Finlandais est passé de la vie dispersée au milieu des bois à celle des villes.

Le système social général pèse sur le système de la propriété forestière non seulement au niveau des structures légales, mais aussi par action directe sur chacun des sylviculteurs qui sont fortement attirés loin de leurs forêts par les emplois des secteurs secondaires et tertiaires.

La vitesse avec laquelle s'effectuera le passage de la propriété de subsistance à la propriété patrimoine dépend donc de l'évolution globale de la société, évolution sur laquelle les responsables forestiers auront à faire des paris raisonnés avant de tracer leur plan d'action. Ils auront en particulier à s"interroger sur la permanence des "valeurs " au sein de la société finlandaise et sur l'influence que pourraient avoir de nouvelles conceptions.

Le fait que le système de la propriété forestière soit dominé par celui de la société finlandaise en général, a comme conséquence que ce sont les valeurs de ce dernier groupe qui l'emportent et l'emporteront. Nous pouvons expliciter la chose en observant que le droit d'aînesse serait peut être le mode de transmission des héritages à retenir si le système social de la propriété forestière était dominant et ceci en raison des avantages du non-fractionnement des massifs aménagés. Le système social général a d'autres valeurs à respecter et impose donc au système dominé le partage égal des forêts entre les enfants.

\section{CHAMP D'ACTION D'UNE POLITIQUE FORESTIĖRE}

Ne reste-t-il pas de champ où le responsable en matière de politique forestière puisse exercer son action? Faut-il qu'il se contente de bâtir tel ou tel modèle de production des forêts sous la dépendance de changements sociaux dans la forêt privée, changements eux-mêmes induits par l'évolution générale de la société?

Non, le responsable a la possibilité d'agir sur le système au niveau des sorties, c'est-à-dire sur le comportement des propriétaires forestiers et sur la commercialisation du bois, par exemple par une réglementation contraignante ou un système d'aides.

Mais cette politique relative à la forêt privée ne sera efficace que s'il est tenu compte en la définissant de l'ensemble des contraintes provenant du système et de la société, et de l'évolution probable de ces contraintes.

\section{APPLICATION DE LA MÉTHOdE A LA FORÊT FINLANDAISE}

L’auteur applique les principes dégagés ci-dessus aux transformations sociales dans la forêt privée finlandaise de 1970 à la fin du siècle.

Différentes vitesses d'urbanisation et différents niveaux de production agricole conduisent à une série de répartitions de la propriété forestière privée, entre la forêt patrimoine et la forêt de subsistance.

L'intervention de l'État et des industriels sur le marché des sols forestiers est également envisagée en fonction de l'évolution générale du pays. L'auteur pense que la masse des transferts entre la propriété privée - forêts patrimoines et forêts de subsistance réunies - et la forêt industrielle ou d'État risque d'être si importante qu'une régulation pourrait être imposée par l'ensemble du système social s'exprimant à travers la législation. 


\section{OBSERVATIONS}

Une application de ces procédés de réflexion au cas de notre pays paraît présenter un intérêt considérable.

L'observation qui fait du système social général un ensemble dominant par rapport au système propre de la propriété particulière souligne l'importance des études engagées sur la structure de la propriété forestière. Elle doit également rendre vigilant le responsable forestier dont les efforts sont sans cesse menacés par l'évolution d'un corps social sur lequel il n'a guère de prise, mais auquel il doit s'adapter.

Tous ceux qui ont travaillé à la mise sur pied de groupements forestiers de petits propriétaires voisins savent bien combien il est difficile d'agir sur la structure de la propriété.

Cependant, et cette observation n'enlève rien à l'estime que suscite ce travail, on ne saurait, sans discussion, étendre au cas de notre pays les présupposés de l'étude de $M$. Reunala. La forêt de subsistance ne nous semble pas un « état " intrinsèquement supérieur à la forêt patrimoine du point de vue de la "valeur " que constitue l'activité économique générale du pays.

Ceci est peut être vrai pour la Finlande où les boisements sont relativement proches de l'optimum technique permis par le climat. En France, au contraire, la production de la forêt est très loin de cet état optimal. Un effort considérable d'investissement est nécessaire pour améliorer la qualité des peuplements.

Qui, dans notre pays est prêt à faire les sacrifices nécessaires à l'amélioration des massifs ? Peut-on compter sur un effort à grande échelle de la puissance publique puisant dans les ressources des contribuables? L'état d'esprit si peu forestier de l'ensemble du corps électoral français en fait douter.

En réalité ce sont les propriétaires pour qui les bois constituent un patrimoine qui détiennent les moyens de cette amélioration. Ils peuvent en effet consacrer à la rénovation de leurs peuplements une partie du surplus de leur activité principale.

Nous voyons bien qu'ils s'y emploient chaque fois qu'on leur en donne la possibilité, contribuant ainsi à la transformation de la fraction majoritaire de la forêt finlandaise.

$\mathrm{Si}$ les autorités finlandaises adaptent leur politique à cette catégorie sociale nouvelle pour eux, ils obtiendront les larges résultats que nous connaissons souvent ici où l'évolution s'est réalisée dans le même sens il y a déjà plusieurs générations.

Condensé par :

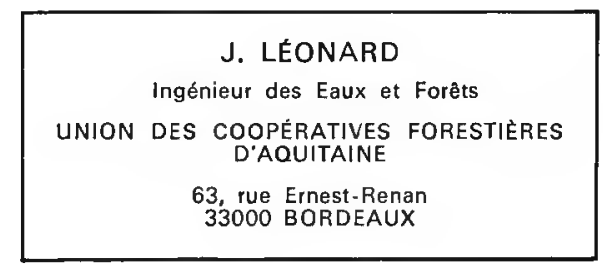

